\title{
Reflective and photoacoustic infrared spectroscopic techniques in assessment of binding media in paintings
}

\author{
Tomasz Łojewski • Jacek Bagniuk • Andrzej Kołodziej • \\ Joanna Łojewska
}

Received: 2 February 2011 / Accepted: 14 June 2011 / Published online: 29 July 2011

(c) The Author(s) 2011. This article is published with open access at Springerlink.com

\begin{abstract}
This study proposes a method to estimate the lipid content in binding media in paintings that can be used at any laboratory equipped with an infrared spectrometer. The lipid content estimator, termed greasiness index (GI), is defined as a ratio of lipid $\nu(\mathrm{C}=\mathrm{O})$ and protein amide $\mathrm{I}$ bands at 1743 and $1635 \mathrm{~cm}^{-1}$, respectively. Three Fourier transform infrared (FTIR) sampling techniques were evaluated for GI determination: reflective attenuated total reflection-ATR, specular reflection microscopy- $\mu \mathrm{SR}$ and photoacousticPAS. A set of model painting samples containing three tempera binding media (casein, egg, egg + oil), seven pigments and one varnish type were used in the study. Multivariate analysis was used to evaluate the resulting data. A good reproducibility of GI was obtained by ATR and PAS but not with $\mu \mathrm{SR}$. The discriminative power of the technique is higher for unvarnished samples, but, generally, the GI estimator can be used for the categorisation of binding media in large populations of painting samples analysed with the same FTIR technique (sampling technique, detection, etc.).
\end{abstract}

\section{Introduction}

The study of heritage objects presents a number of challenges for the researcher dealing with conservation chemistry: sample complexity, poorly known provenance of samples and limitations upon sample collection. This final point

T. Łojewski (₫) · J. Bagniuk · J. Łojewska

Faculty of Chemistry, Jagiellonian University, Ingardena 3, 30-060 Krakow, Poland

e-mail: lojewski@chemia.uj.edu.pl

\section{A. Kołodziej}

Opole University of Technology, Faculty of Civil Engineering, Katowicka 48, 45-061 Opole, Poland arises from the fact that, to be applied to a heritage object, the analytical method of choice must be non-invasive, or at worst micro-destructive. Through the application of instrumental physicochemical analysis, a heritage scientist is better able to inform the conservation treatment of the objects, and thereby the objects are better preserved for the future. Following this idea, this paper aims at evaluating the existing Fourier transform infrared (FTIR) techniques for the analyses of paintings and at elaborating a simple quantitative method for the assessment of the lipid content in painting binding media.

While the analysis of inorganic materials, usually in the form of small particles of pigments or fillers, included in paintings is relatively straightforward, organic mixtures in binding media and in varnishes still remain a vexing issue for analytical chemistry $[1,2]$. This has been a direct motivation for our studies. Traditional binding media, as used by artists, can be categorised into four general classes of organic compounds: proteins, lipids, polysaccharides and resins [1, 3]. The knowledge of the lipid content, hereafter referred to as greasiness index (GI), in particular, can help conservators to recognise the material in binding media, in order to plan the necessary restoration treatments and to detect previous ones. It may also be of help to art historians in any studies of painting techniques.

A great deal has been published about the application of FTIR (and complementary Raman) spectroscopies to the study of paintings and within this area also of binding media. Several literature reviews of the topic have been issued by Casadio and Toniolo [4] as well as by Weerd et al. [5] and by Prati et al. [6]. The majority of publications, however, focus on single-object case studies, for example in [5, 7-10]. More general approaches can be found in Refs. [2, 6, 7, 9, 11]. These studies emphasise the applications of the microattenuated total reflection (ATR) technique to the analyses 
of the cross sections of paintings [5-7, 11, 12], or fibre optics (FO) based methods, which are more complex to interpret $[2,9]$. The assignments of the bands of the binding media and pigments can be found in [3, 9, 12]. Mazzeo et al. [12] and Ploeger et al. [9] proposed the band assignment in the spectra of various binding media and in particular the bands coming from lipid $(\mathrm{C}=\mathrm{O}$ vibrations in ester) and protein $(\mathrm{C}=\mathrm{O}$ in amide $\mathrm{I}$ and $\mathrm{N}-\mathrm{H}$ in amide $\mathrm{II})$. The alterations of binding media due to the interactions with pigments are discussed in $[7,9,12]$. Ploeger et al. identified the changes in the band frequencies between sampling techniques [9]. In the cited work, an interesting approach is the use of the Kramers-Kronig equation to correct the intensities of the spectra obtained with specular reflection microscopy ( $\mu$ SR). A comprehensive survey of organic compounds (also binding media) included in original paintings was presented by Rosi et al. [2]. Rosi et al. [2] used non-invasive fibre optics reflectance FTIR measurements of original art objects with cluster analysis on selected marker bands to resolve the complex spectra obtained with this technique and to differentiate between the organic ingredients present in various samples. In the survey by Jurado-López and de Castro [13], principal component analysis (PCA) was applied for the assignment of $\mathrm{C}-\mathrm{H}$ stretching overtones. The analysis is based on different aliphatic/saturated versus unsaturated aromatic profiles which give rise to significant differences in the $\mathrm{C}-\mathrm{H}$ stretching region. The application of the second principal component (PC2) related to greasiness of those media allowed them to sort out their spectra by increasing lipid content. Though chemometry is a powerful tool to approach the problem of quantitative analysis of binding media in the samples, it needs, as the authors claim, further investigation. A chemometric model, to be informative, requires a huge number of samples to be gathered in the spectral library.

In this study, we aim to show a very simple quantitative approach for a rough evaluation of binding media in painting samples that can be performed by any laboratory equipped with a FTIR spectrometer fitted with one of the reflectance measuring devices. Amongst the sampling techniques available for FTIR spectroscopy such as diffuse reflectance-DRIFT, specular reflection-SR, fibre optics reflectance-FOR, attenuated total reflectance-ATR and photoacoustic-PAS, only the microscopic ( $\mu$ SR and $\mu$ ATR) and fibre optics (FO) techniques meet the conservators' requirements for non-invasiveness. In our study, the ATR technique has been chosen as a model to emulate capabilities of ATR microscopy ( $\mu$ ATR).

The evaluation of PAS applicability to typical analytical tasks connected with painting characterisation is the second goal of this study. The application of the PAS technique to cultural heritage objects is very limited, as summarised in the review by Casadio and Toniolo [4], which is rather surprising as PAS is a routinely used tool in scientific and industrial laboratories [14]. From the point of view of the mechanism of formation of the photoacoustic signal, the PAS technique has many advantages over traditional and reflectance FTIR sampling techniques, such as no sample preparation, applicability to highly absorbing samples, reproducible baseline, the signal is representative of the bulk of the sample and finally the opportunity of performing depth profiling with a step-scan FTIR spectrometer $[14,15]$. The only obstacle that prevents its broader application in conservation science is the lack of the technical solutions which would allow for the non-invasive examination of original paintings, but new laser-based detection systems described in the literature [14] open new prospects for the application of PAS in conservation science.

\section{Experimental}

\subsection{Materials}

A set of model painting samples used for this study was prepared using three tempera binding media and seven pigments described in Fig. 1. Malachite pigment was purchased as a mineral and prepared by grinding and sieving. Six other pigments were: viridian, French ultramarine, yellow ochre, terre verte, burnt and raw umber-all obtained from Winsor \& Newton. The criterion for choice of the pigment set was that they should appear in historical paintings and vary

\begin{tabular}{|c|c|c|c|c|c|}
\hline \multirow{2}{*}{$\begin{array}{l}\text { column } \\
\text { row }\end{array}$} & $\mathbf{A}$ & B & C & & \\
\hline & 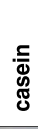 & $\begin{array}{l}\text { 영 } \\
\text { o } \\
\frac{0}{0} \\
\frac{0}{3}\end{array}$ & 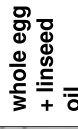 & & \\
\hline 1 & & & & no pigment & - \\
\hline 2 & & & & malachite & $\mathrm{Cu}_{2} \mathrm{CO}_{3}(\mathrm{OH})_{2}$ \\
\hline 3 & & & & viridian & $\mathrm{Cr}_{2} \mathrm{O}_{3} \cdot 2 \mathrm{H}_{2} \mathrm{O}$ \\
\hline 4 & & & & ultramarine & $\mathrm{S}_{3}{ }^{-}$in lapis lazuli \\
\hline 5 & & & & $\begin{array}{l}\text { yellow } \\
\text { ochre }\end{array}$ & $\mathrm{FeO}(\mathrm{OH})$ \\
\hline 6 & & & & terre verte & $\mathrm{FeSiO}_{3}$ in glauconite \\
\hline 7 & & & & $\begin{array}{l}\text { burnt } \\
\text { umber }\end{array}$ & $\mathrm{Fe}_{2} \mathrm{O}_{3}+\mathrm{MnO}_{2}$ in clay \\
\hline 8 & & & & raw umber & $\alpha-\mathrm{FeOOH}$ In clay \\
\hline
\end{tabular}

Fig. 1 Model painting sample compositions. Columns (A, B, C): binding media and rows (1-8): pigment name and simplified chemical formula 
sufficiently in chemical composition (Fig. 1) to be able to validate the analytical method for lipid content assessment.

The binding media were prepared using eggs from a local grocery and materials obtained from Kremer Pigmente: casein (cat. no. 63200), ammonium carbonate (64021) and linseed oil (73050). Also, rabbit skin glue (KREMER, cat. no. 63025) and calcium carbonate p.a. (Polskie Odczynniki Chemiczne) were used for the support preparation and dammar resin (KREMER, cat. no. 79305) for varnishing.

\subsection{Sample preparation}

Three binding media were prepared according to the recipes found in [16]: casein tempera (A), whole egg tempera (B) and greasy whole egg tempera $(\mathrm{C})$. Casein tempera was prepared by mixing casein and ammonium carbonate solutions. To obtain it, $20.0 \mathrm{~g}$ of casein was mixed with $100 \mathrm{~cm}^{3}$ of water at $30^{\circ} \mathrm{C}$. To the suspension, a concentrated solution of $\left(\mathrm{NH}_{4}\right)_{2} \mathrm{CO}_{3}$ was added and intensely stirred to obtain a homogeneous emulsion. Whole egg tempera was obtained from stirred egg and water with a volumetric proportion of $2: 1$. To produce the emulsion, the mixture was poured into a bottle and then shaken. Whole egg and linseed oil tempera was prepared as above using a mixture of oilwhole eggwater with a proportion of 1:1:1. However, the first two ingredients were mixed by shaking prior to addition of water.

The pigments were ground with a given binding medium until the mixture looked homogeneous. Pigment-binder ratios were not noted since different pigments demanded different amounts of binding medium to form a usable paint. Half of each sample area was varnished after 6 months of storage. Spectra were collected after approximately an additional 6 months of storage.

Figure 1 shows a part of a canvas where the samples used for this study were painted as well as the corresponding symbols for their identification. The model painting samples were prepared in the form of a matrix made of rectangles painted on canvas. In order to obtain statistical heterogeneity of the layered material, the different layers were not distributed evenly. Each rectangle was $8 \mathrm{~cm} \times 3 \mathrm{~cm}$ and half of this area was varnished. The canvas was previously primed according to guidelines from [16]. In order to reduce the number of ingredients for the study, only rabbit glue, calcium carbonate and distilled water were used in a priming layer.

Prior to measurements, small discs, $5 \mathrm{~mm}$ in diameter, were cut out from each sample. The same disc was used for measurements with all three sampling techniques, which were used in a sequence which is as follows: $\mu$ SR and PAS (non-invasive) and then ATR. The ATR measurements were performed last, because of the partial damage caused to the samples when they are pressed to the surface of the ATR crystal.

\subsection{Instrumentation}

Spectra were collected using a Thermo/Nicolet FTIR spectrometer equipped with a Thermo-Nicolet Centaur $\mu$ s infrared microscope, attenuated total reflection (ATR) and photoacoustic (PAS) devices. The ATR measurements were done using a Specac Golden Gate ATR accessory with diamond crystal and $\mathrm{ZnSe}$ lenses. ATR spectra were collected with $4 \mathrm{~cm}^{-1}$ resolution and averaged with 64 scans. The microscopic measurements were done using a ThermoNicolet Centaur $\mu$ s infrared microscope working in $8^{\circ}$ reflection mode attached to the spectrometer. The $\mu$ SR spectra were collected with $4 \mathrm{~cm}^{-1}$ resolution and averaged with 64 scans. The PAS measurements were done using an MTEC Photoacoustic model 300 accessory. PAS spectra were collected with $8 \mathrm{~cm}^{-1}$ resolution and averaged with 256 scans. For the ATR and $\mu$ SR techniques a MCT detector was used, while for PAS a built-in microphone was used as the detector.

Minimum processing was done to the spectra collected with the various techniques. The ATR spectra were processed with an automatic ATR correction which adjusts for the wavelength-dependent depth of the signal generation. From each PAS spectrum the spectrum of water vapour was subtracted with a factor obtained upon minimising the integral of the squared second derivative of the spectra after subtraction.

\section{Results and discussion}

Tempera binding media are often classified by artists and conservators as 'more' and 'less' greasy. Although there is no precise definition of greasiness, it can certainly be linked to lipid-protein relative concentration in binding media. Thus, we define a greasiness index (GI) as a ratio between absorbance values or integrated absorbance values of lipid $v(\mathrm{C}=\mathrm{O})$ (in ester bond) and protein amide I (carbonylic group in the peptide bond) bands, which appear at around $1743 \mathrm{~cm}^{-1}$ and $1635 \mathrm{~cm}^{-1}$, respectively (Figs. 2 and 3).

The choice of marker bands seems to be natural, since they are strong features in the infrared spectra of the painting samples as can be inferred from Fig. 2. The figure shows exemplary spectra collected by ATR, $\mu$ SR and PAS techniques for the reference samples (no pigment—row 1) with three various binding media (A1-C1 in Fig. 1), and also for the malachite (A2-C2) and ochre (A5-C5) containing samples. Table 1 summarises the positions of the marker bands in the reference samples $(\mathrm{A} 1-\mathrm{C} 1)$ for the three techniques used.

When analysing the frequencies in Table 1 , it can be inferred that the bands' positions from the lipidic $\mathrm{C}=\mathrm{O}$ group 

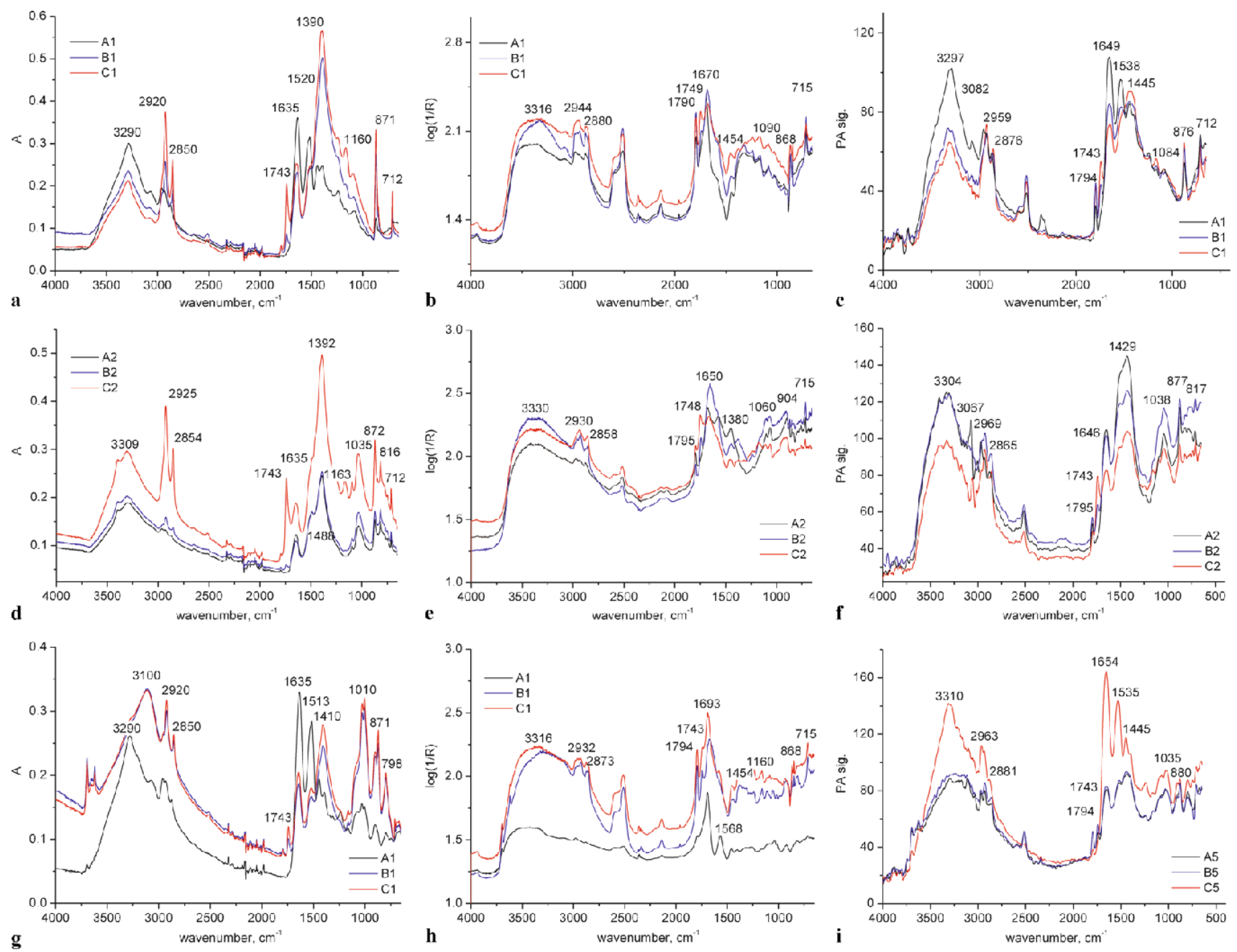

Fig. 2 Comparison of FTIR spectra collected by ATR (a, d, g), $\mu$ SR $(\mathbf{b}, \mathbf{e}, \mathbf{h})$ and PAS $(\mathbf{c}, \mathbf{f}, \mathbf{i})$ techniques for the samples described in Fig. 1: the reference samples with three binding media (A1, B1, C1) with no pigment $(\mathbf{a}-\mathbf{c})$; the malachite samples $(\mathbf{d}-\mathbf{f})$ with three binding media (A2, B2, C2); the yellow ochre samples $(\mathbf{g}-\mathbf{i})$ with three binding media (A5, B5, C5)

Table 1 The positions of the marker bands in the reference samples A1, B1, C1 (Fig. 1) as measured by the three FTIR techniques

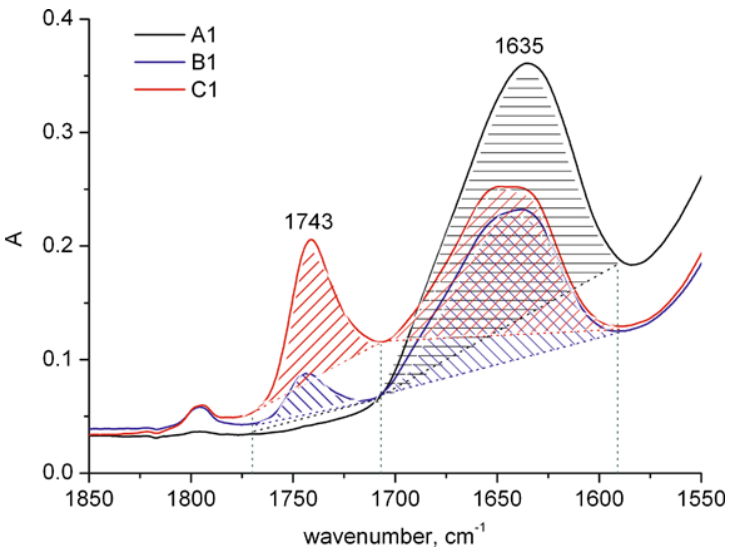

Fig. 3 The integration method of the marker bands from vibrations of $\mathrm{C}=\mathrm{O}$ groups in ester (lipid) at $1743 \mathrm{~cm}^{-1}$ and $\mathrm{C}=\mathrm{O}$ groups in amide I (peptide) at $1635 \mathrm{~cm}^{-1}$

\begin{tabular}{|c|c|c|c|c|c|c|}
\hline \multirow{3}{*}{$\begin{array}{l}\text { Reference } \\
\text { sample }\end{array}$} & \multicolumn{6}{|c|}{ Marker band position, $\mathrm{cm}^{-1}$} \\
\hline & \multicolumn{3}{|c|}{ Lipid $\mathrm{C}=\mathrm{O}$} & \multicolumn{3}{|c|}{ Amide I } \\
\hline & ATR & $\mu \mathrm{SR}$ & PAS & ATR & $\mu \mathrm{SR}$ & PAS \\
\hline $\mathrm{A} 1$ & - & - & - & 1635 & 1690 & 1649 \\
\hline B1 & 1743 & 1747 & 1743 & 1636 & 1671 & 1650 \\
\hline $\mathrm{C} 1$ & 1741 & 1749 & 1742 & 1638 & 1670 & 1649 \\
\hline
\end{tabular}

and the amide I group vary with sample composition and with the technique used. The first can be accounted for by the interactions of pigments with binding media. These interactions, especially with respect to lipids, can eventually lead to the formation of fatty acid salts (soaps) whose formation was noted in $[7,12]$. The latter can be explained by the differences in the mechanisms of signal formation 
in the studied techniques. The responses come from various depths of the samples, which roughly follow the order: $\mu \mathrm{SR}<\mathrm{ATR}<\mathrm{PAS}$. While $\mu \mathrm{SR}$ can be treated as a more surface-sensitive method responding directly to the surface texture and morphology, the PAS signal represents bulk composition, which can be explained by the mechanism of propagation of thermal waves through analysed material. The surface sensitivity of $\mu$ SR can be illustrated by the appearance of bands invisible with other methods (bands in the range $2700-2000 \mathrm{~cm}^{-1}$ in Fig. $\left.2 \mathrm{~b}, \mathrm{e}, \mathrm{h}\right)$. The sensitivity of $\mu \mathrm{SR}$ is also rather low when compared to the other techniques used; this is due to the little energy reflected by a sample that reaches the detector. The apparent shifts in bands' positions observed in the $\mu$ SR spectra can be explained by the optical effects connected with specular reflection (described by the Kramers-Kronig equation) [9].

For all reasons given above, the resolution and repeatability of the spectra collected by $\mu$ SR are low compared to the ATR and PAS methods. This is why the GI values for the $\mu$ SR spectra will be calculated from the marker bands' intensities: for the lipid $\mathrm{C}=\mathrm{O}$ band at $1749 \mathrm{~cm}^{-1}$ and for the amide $\mathrm{I}$ band at $1670 \mathrm{~cm}^{-1}$, which are arbitrarily chosen but then consequently used (see $\mu$ SR for varnished samples in Fig. 7).

The integration method for the GI calculation from the ATR and PAS spectra is presented in Fig. 3 for the reference samples (A1-C1) given as an example. The integration was performed using three-point baseline correction for each spectrum (obtained with ATR and PAS techniques). Thus, if there is no band at around $1743 \mathrm{~cm}^{-1}$ representing lipid content in binding media the GI can be equal to zero or even lower than zero: in such a case the baseline (dotted black line) appears above the integrated curve (curve A1). For convenience, the spectra presented in the next figures have been normalised to the amide I band area (calculated in the range from 1707 to $1591 \mathrm{~cm}^{-1}$ ) so that the GI values could be directly assessed from the intensity of the $1743 \mathrm{~cm}^{-1}$ band.

The key question to answer is whether the GI defined in the way presented above and measured with various tech- niques is representative of the actual lipid to protein ratio. It seems that there are three cases when the information from the GI can be obscure: (i) low signal reflection thus giving saturated spectra, (ii) strong deformation of marker bands and their overlapping with bands coming from other painting materials such as carbonates that are often present in pigments [7] and (iii) spatially heterogeneous samples, particularly multilayered samples [5]. These issues will be demonstrated and discussed using various examples presented in the next passages. To answer the question regarding the GI reliability for the determination of the lipid content in the binding media, the performance criteria, for a certain technique, including: the response range, the precision (repeatability of the GI), the specificity and, finally, the discriminative power, will be tested.

The three binding media used for this study set the range within which the GI values can appear. As can be noted in Fig. 4, the GI values show the expected growing tendency of the standardised $1743 \mathrm{~cm}^{-1}$ band intensity with the increasing lipid content of the binding medium (casein $<$ egg $<$ egg + oil). The growing tendencies with the increasing lipid content can also be noted for the GI values presented as histograms in Figs. 5, 6 and 8 (compare the group of three bars for each measurement). The GI values can be less than or equal to zero for the samples denoted as A that contain only casein (no lipid) when calculated using the integration method from the ATR and PAS spectra. Due to the difference in the calculation of the GI from the $\mu$ SR spectra, the values are positive even though samples A do not contain any lipid.

An important issue to discuss is the repeatability of the GI values. The results are presented in Fig. 5 in the form of histograms. To verify the repeatability, the spectra were collected for five randomly cut samples from the fifth row of the sample matrix (A5, B5, C5 containing yellow ochre) (Fig. 1). The procedure was repeated for each technique. In this way, the five GI values obtained for a given binding medium (A, B, C) and for a given technique (Fig. 5(a), (b), (c)) were averaged. We may assume for the purpose of binding media rough evaluation that the GI es-
Fig. 4 Standardised ATR (a), $\mu \mathrm{SR}(\mathbf{b})$ and PAS (c) spectra of $\mathrm{A} 1, \mathrm{~B} 1, \mathrm{C} 1$ samples as described in Fig. 1. The ATR and PAS spectra were normalised to the area of the amide I band and the $\mu \mathrm{SR}$ spectra to the maximum of the same band
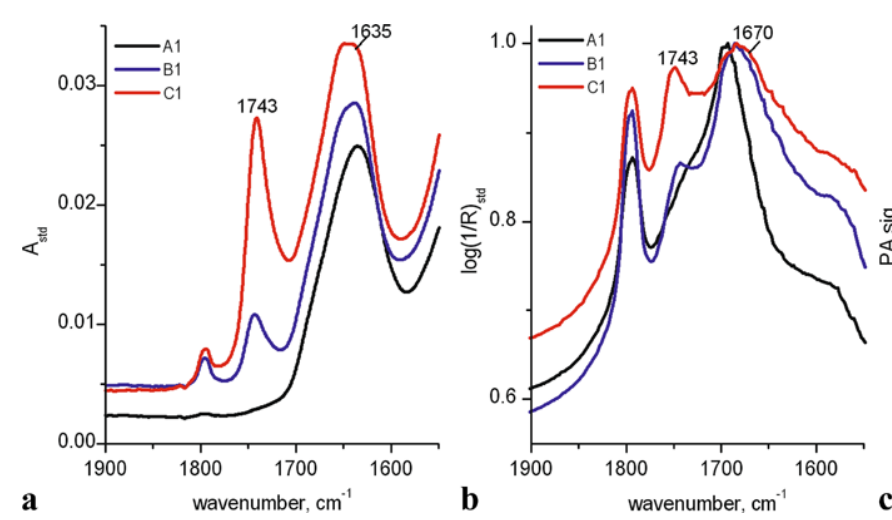
Fig. 5 GI values obtained for five disc samples taken from A5, B5, C5 samples as described in Fig. 1. The last group of bars presents average GI values and their standard deviations. Disc number is a sample chosen for analysis from a certain rectangle in the matrix presented in Fig. 1
Fig. 6 GI values obtained for all pigment-containing samples (\# = row numbering as in Fig. 1). The last group of bars presents average GI values and their standard deviations. Cross symbol by the top of a bar shows outliers excluded from calculation of GI average. Row number refers to pigment type as in Fig. 1
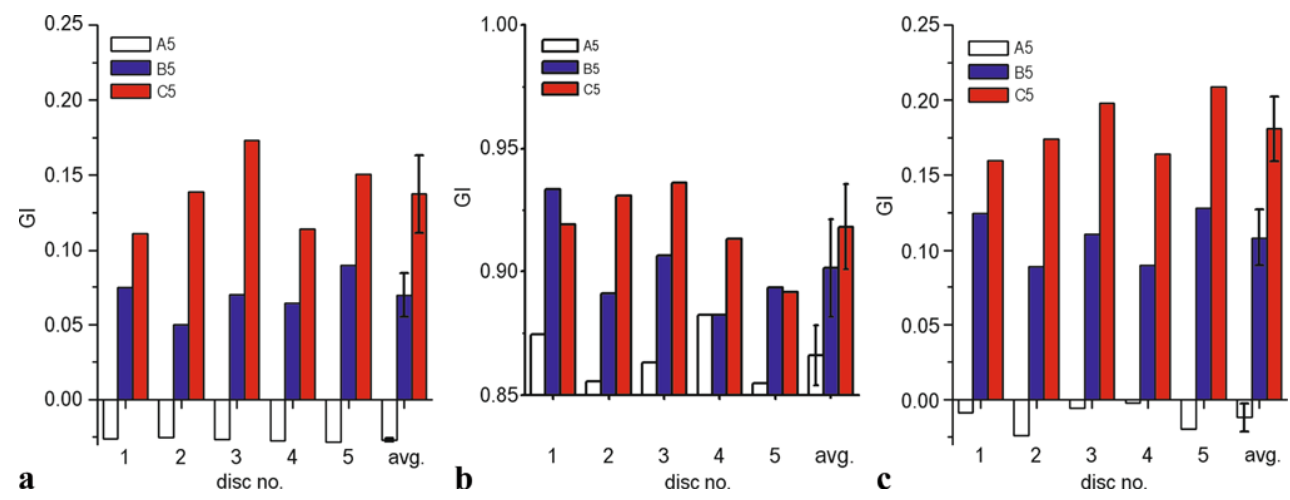
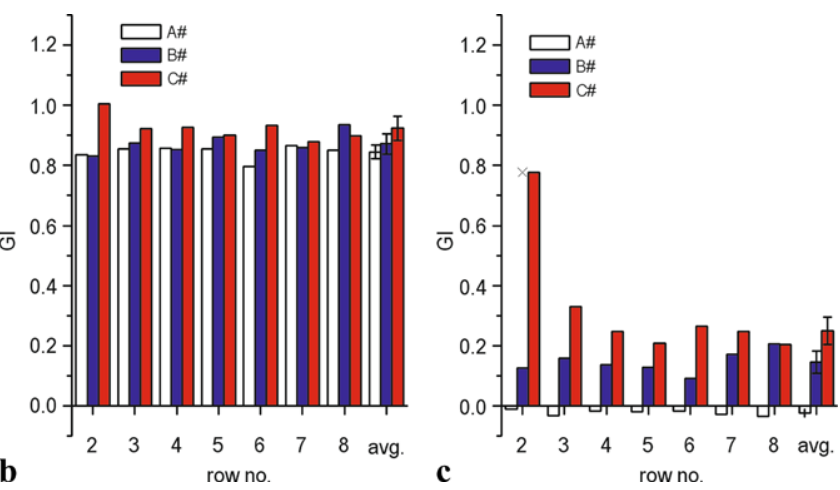

Fig. 7 ATR (a), $\mu$ SR (b) and PAS (c) spectra of varnished samples A5, B5, C5

( $\mathrm{v}$ - varnish) as described in Fig. 1. The spectra were scaled to intensity of the band at $1705 \mathrm{~cm}^{-1}$ ( $\mathrm{C}=\mathrm{O}$ in varnish). The inserted numbers refer to absorbance values at wavenumbers 1743 and $1635 \mathrm{~cm}^{-1}$ (maxima of lipid $\mathrm{C}=\mathrm{O}$ and protein amide $\mathrm{I}$ bands)
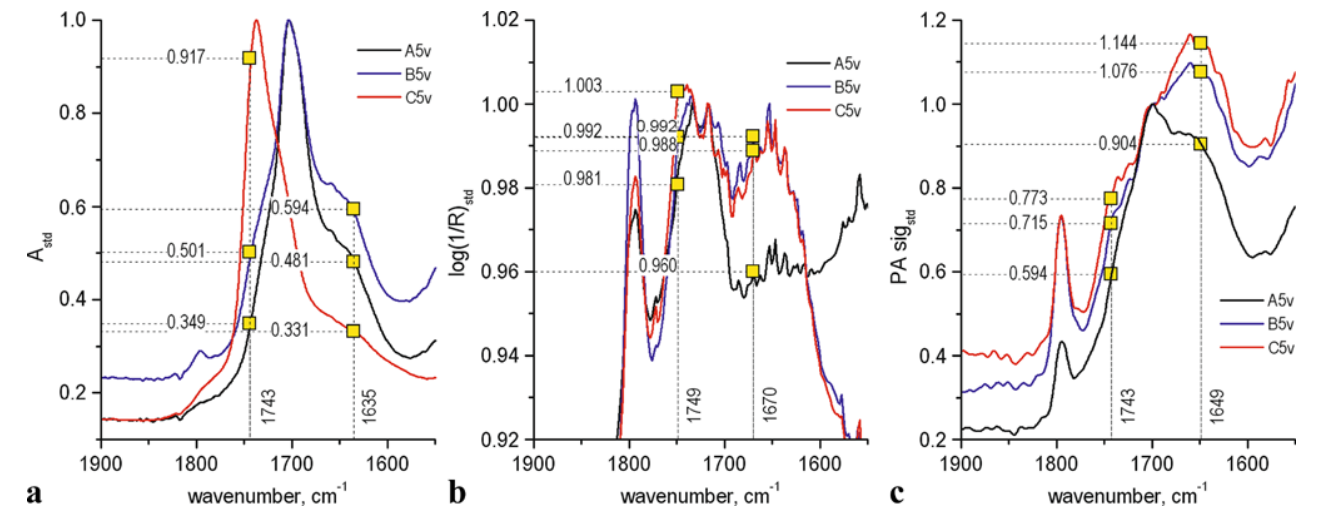

Fig. 8 GI values obtained from varnished samples $(\mathrm{A \# v}, \mathrm{B} \# \mathrm{v}$, $\mathrm{C \# v}$ for rows 2, 3, 5, 6 denoted using symbol \#). The last group of bars presents average GI values and their standard deviations. Row no. refers to pigment type as in Fig. 1
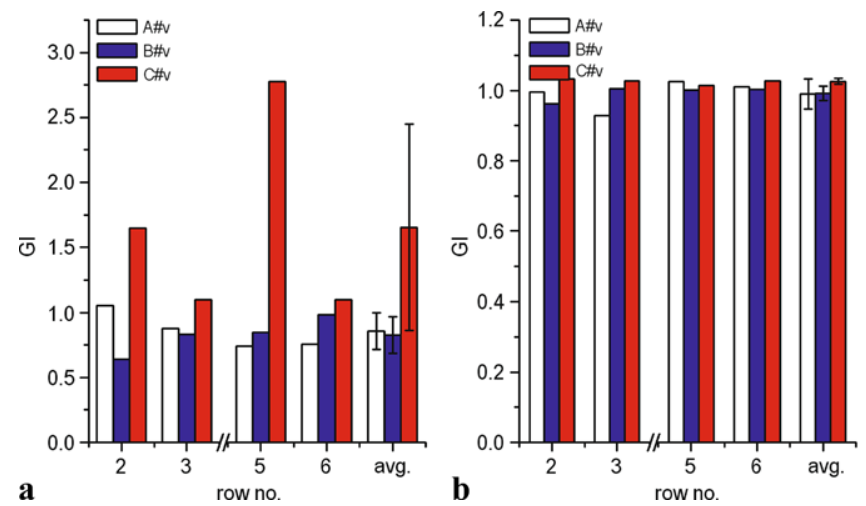
timator is precise if its standard deviation does not exceed $30 \%$ of its average value. In this way, only the result gathered by PAS for the casein sample does not pass the precision test, but this is because the sample does not contain any lipid, which is indicated by the negative value of GI.

In an attempt to check the GI specificity, the spectra were collected for all pigment-containing samples from the 2nd to the 8th rows and the A, B, C columns of the matrix in Fig. 1 . The specificity is understood here as the extent to which it can determine particular analytes in a complex mixture without interference from other components in the mixture. The single and averaged GI values obtained in such a way are presented in Fig. 6. The differences in the average GI values reflect the differences in the lipid content in binding media. The standard percent error exceeds $30 \%$ for the B and C samples measured by ATR.

Proceeding with the evaluation of the GI for the varnished samples, the overall observation is that a varnish layer greatly disturbs the determination of GI. Indeed, varnish contains the band $v(\mathrm{C}=\mathrm{O})$ at $1705 \mathrm{~cm}^{-1}$ which appears in a spectrum as a much more intensive maximum than the marker bands used for GI calculations. This is certainly due to the fact that the varnish layer is located at the surface of the samples (Fig. 7). For this reason, $\mu$ SR spectra are the most affected by the presence of varnish, as can be noted by comparing the spectra obtained with the techniques used in this study. In the convoluted spectrum, the lipid $v(\mathrm{C}=\mathrm{O})$ and amide I bands emerge as shoulders on an overall broad band
(ATR and PAS in Fig. 7a and c, respectively) or disappear inside it, thereby influencing its maximum position ( $\mu$ SR in Fig. 7b); it seems reasonable to calculate the GI values as the ratios of absorbance at positions where lipid and amide bands show maxima in the spectra of the reference unvarnished samples (Fig. 3). The absorbance values corresponding to the wavenumbers of the reference bands are indicated in the spectra presented in Fig. 7. Figure 8 presents GI values calculated in this way for the set of varnished pigmentcontaining samples (rows 2, 3, 5, 6 of the matrix in Fig. 1) as well as their averaged values and standard deviations, similarly to those presented in Fig. 6 . Since the $\mu$ SR technique loses all sensitivity when the varnish layer is applied to the samples, due to high spectral saturation and strong specular reflection, there are no important differences in the GI values among the samples differing in the binding media type. The ATR and PAS techniques struggle with sorting out less greasy media (casein and whole egg), because of the small contribution of the lipid $v(\mathrm{C}=\mathrm{O})$ band to the observed convoluted band. Generally, for the more greasy media, both ATR and PAS techniques will be more sensitive in categorising the samples because of the higher contribution of the lipid $\mathrm{C}=\mathrm{O}$ band.

Table 2 summarises the results of validation of the GI estimator for semiquantitative assessment of binding media presenting the average values and standard deviations for all samples and techniques. The most important performance criterion for the GI estimator is its discriminative power. We
Table 2 Average values of GI from all measurements performed in this study
${ }^{\mathrm{a}} \mathrm{v}$ in the sample name-varnished samples

\begin{tabular}{|c|c|c|c|c|c|c|c|}
\hline \multirow[t]{2}{*}{ Technique } & \multirow{2}{*}{$\begin{array}{l}\text { Measured } \\
\text { samples }^{\mathrm{a}}\end{array}$} & \multirow{2}{*}{$\begin{array}{l}\text { Data for GI } \\
\text { calculation }\end{array}$} & \multicolumn{3}{|c|}{ Greasiness index (GI) } & \multirow[t]{2}{*}{ Parameter 1} & \multirow[t]{2}{*}{ Parameter 2} \\
\hline & & & $\overline{\mathrm{A}}$ & $\mathrm{B}$ & $\mathrm{C}$ & & \\
\hline \multirow[t]{6}{*}{ ATR } & A5-C5 & Band areas & -0.0268 & 0.070 & 0.137 & 0.16 & 0.59 \\
\hline & five discs & & \pm 0.0012 & \pm 0.014 & \pm 0.026 & & \\
\hline & $\mathrm{A} 2-\mathrm{C} 8$ & Band areas & -0.0313 & 0.080 & 0.202 & 0.32 & 0.88 \\
\hline & & & \pm 0.0048 & \pm 0.031 & \pm 0.076 & & \\
\hline & $\mathrm{A} 2 \mathrm{v}-\mathrm{C} 3 \mathrm{v}$ & Max absorbance & 0.86 & 0.83 & 1.65 & -9.33 & 1.13 \\
\hline & $\mathrm{A} 5 \mathrm{v}-\mathrm{C} 6 \mathrm{v}$ & & \pm 0.14 & \pm 0.14 & \pm 0.79 & & \\
\hline \multirow[t]{6}{*}{$\mu \mathrm{SR}$} & A5-C5 & Band heights & 0.866 & 0.899 & 0.919 & 0.96 & 1.67 \\
\hline & five discs & & \pm 0.012 & \pm 0.019 & \pm 0.014 & & \\
\hline & $\mathrm{A} 2-\mathrm{C} 8$ & Band heights & 0.845 & 0.871 & 0.924 & 2.19 & 1.43 \\
\hline & & & \pm 0.023 & 0.035 & 0.040 & & \\
\hline & $\mathrm{A} 2 \mathrm{v}-\mathrm{C} 3 \mathrm{v}$ & Max absorbance & 0.990 & 0.992 & 1.0254 & 24.03 & 0.87 \\
\hline & $\mathrm{A} 5 \mathrm{v}-\mathrm{C} 6 \mathrm{v}$ & & \pm 0.043 & \pm 0.021 & \pm 0.0081 & & \\
\hline \multirow[t]{6}{*}{ PAS } & A5-C5 & Band areas & -0.0120 & 0.108 & 0.181 & 0.23 & 0.56 \\
\hline & five discs & & \pm 0.0092 & \pm 0.019 & \pm 0.022 & & \\
\hline & $\mathrm{A} 2-\mathrm{C} 8$ & Band areas & -0.0231 & 0.145 & 0.250 & 0.27 & 0.79 \\
\hline & & & \pm 0.0090 & \pm 0.037 & \pm 0.045 & & \\
\hline & $\mathrm{A} 2 \mathrm{v}-\mathrm{C} 3 \mathrm{v}$ & Max absorbance & 0.618 & 0.657 & 0.79 & 2.24 & 1.36 \\
\hline & $\mathrm{A} 5 \mathrm{v}-\mathrm{C} 6 \mathrm{v}$ & & \pm 0.059 & \pm 0.030 & \pm 0.16 & & \\
\hline
\end{tabular}


may assume that the GI (derived by a certain FTIR technique) can discriminate between two binding media when the differences between averaged GI values of the samples containing different binding media (casein (A5) and egg (B5) or egg + oil (C5)) are substantially higher than the sums of their standard deviations: $\left[\delta_{\mathrm{GI}}(\mathrm{A} 5)+\delta_{\mathrm{GI}}(\mathrm{B} 5)\right]$ or $\left[\delta_{\mathrm{GI}}(\mathrm{B} 5)+\delta_{\mathrm{GI}}(\mathrm{C} 5)\right]$. To express it in a qualitative way, two parameters (denoted as Par 1 and Par 2) are proposed, which are the ratios between the sums of standard deviations and the differences between GI values for pairs of casein (A) and egg (B), as well as egg (B) and egg + oil (C) temperas, respectively:

$\operatorname{Par} 1=\frac{\delta_{\mathrm{GI}}(\mathrm{A})+\delta_{\mathrm{GI}}(\mathrm{B})}{\overline{\mathrm{GI}}(\mathrm{B})-\overline{\mathrm{GI}}(\mathrm{A})}$,

$\operatorname{Par} 2=\frac{\delta_{\mathrm{GI}}(\mathrm{B})+\delta_{\mathrm{GI}}(\mathrm{C})}{\overline{\mathrm{GI}}(\mathrm{C})-\overline{\mathrm{GI}}(\mathrm{B})}$.

The parameters are the mathematical representation of the evaluation of the histograms with standard deviations presented in Figs. 5, 6 and 8. Values in the range from 0 to 1 indicate that for a given set of samples (also the technique involved and the method of GI calculation), the discrimination of the binding media GI is reliable-the smaller the ratio the higher the reliability. Values larger than 1 indicate poor reliability of such discrimination, and no possibility of recognising the same samples. When the value of the ratio is below 0 , the estimation of greasiness using GI fails.

In this way, the ATR and PAS techniques can be considered as discriminative for unvarnished samples, unlike $\mu$ SR, for which in most cases the parameters are higher than 1. For the varnished samples, the GI discriminative power is much less than with unvarnished samples: the values of the parameters are less than 0 or much higher than 1 , except for the PAS technique, which shows slightly higher discriminative power than the other techniques. This can be connected with the fact that the PAS signal is more representative of the sample bulk and not a varnished surface.

The results may suggest that the absolute GI values are dependent on the instrumentation used. Since there are a great many controllable and uncontrollable parameters which contribute to the observed values of GI, its role seems to be more to differentiate the binding media within the population of samples in terms of their greasiness following exactly the same analytical procedure, rather than to provide absolute lipid content.

To place the discussion in a broader context, in the application of the GI estimator to assess real artefacts, except for the $\mu$ SR technique, which does not provide any satisfactory results, the ATR and PAS techniques applied in this study could not be used for real object analyses without certain modifications. With the ATR accessory applied by us, except for the necessity for relatively big samples (at least
$1 \times 1 \mathrm{~mm}^{2}$ ), the problem is that they are locally damaged because of the high pressure exerted on them by the sample vice. Additionally, in real situations for the samples containing several thin layers of paints differing in greasiness, the observed GI reflects average greasiness of all of these layers. The $\mu$ ATR technique, which is routinely applied for the analyses of originals in a micro-destructive mode, can be expected to produce similar results to those obtained using the macro-ATR accessory used for this study. What is more, measuring GI in painting cross sections would permit the determination of the greasiness of each paint layer as well as the avoidance of interferences from the varnish. The commercially available PAS accessories for solid samples are all equipped with sample cups, which need to be closed in a sample compartment during analysis and do not allow precise sample manipulation. That limits the usefulness of the existing technical solutions in the field of conservation science, but promising results obtained in this study together with other advantages of this technique, which were emphasised in the introduction, should further stimulate researchers and equipment manufacturers to produce more suitable devices.

\section{Conclusions}

This paper poses an approach to the application of FTIR spectroscopy to quantitative analysis of binding media in originals of paintings. The greasiness index (GI) proposed in this work and defined as a ratio between intensities of lipid $v(\mathrm{C}=\mathrm{O})$ at $1743 \mathrm{~cm}^{-1}$ and protein amide $\mathrm{I}$ at $1635 \mathrm{~cm}^{-1}$ bands allows semiquantitative estimation of the lipid content in a binding medium. The index is dedicated to discriminative analyses in large populations of samples of classical paintings manufactured from natural materials. Attempts of any absolute assessment of single sample binding medium greasiness are not recommended. The greasiness index gives the most reliable results for the painting samples made of a single layer of paint. The estimation of greasiness in multilayered paintings as well as in varnished ones needs further validation.

The ATR and PAS techniques, although they do not meet the requirement of non-invasiveness emphasised by the conservators, give the most reproducible results of GI. Any technique based on specular reflection will fail in GI evaluation, due to spectral saturation and specular reflection effects (restrahlen and derivative-like bands), which affect the positions and intensities of the marker bands. The results suggest that ATR microscopy should overcome the above-mentioned drawbacks that are shown by ATR and $\mu$ SR alone. Painting cross-section analysis can eliminate the varnish problem and make it possible to determine greasiness of deep painting layers. 
Acknowledgements The study was supported by the grant SPB K/PMN/000023 from the Polish Ministry of Science and Higher Education within the COST action D42 and partly by Cultural Exchange Funds within the Norwegian Financial Mechanism, 11/03/2009/FWK (2009-2011), led by Wawel Royal Castle, National Art Collection.

Open Access This article is distributed under the terms of the Creative Commons Attribution Noncommercial License which permits any noncommercial use, distribution, and reproduction in any medium, provided the original author(s) and source are credited.

\section{References}

1. R. Mazzeo, A. Roda, Anal. Bioanal. Chem. 392, 27-28 (2008)

2. F. Rosi, A. Daveri, C. Miliani, G. Verri, P. Benedetti, F. Piqué, B.G. Brunetti, A. Sgamellotti, Anal. Bioanal. Chem. 395, 20972106 (2009)

3. P. Vandenabeele, B. Wehling, L. Moens, H. Edwards, M. De Reu, G. Van Hooydonk, Anal. Chim. Acta 407, 261-274 (2000)

4. F. Casadio, L. Toniolo, J. Cult. Heritage 2, 71-78 (2001)

5. J. Weerd, R.M.A. Heeren, J.J. Boon, Stud. Conserv. 49, 193-211 (2004)

6. S. Prati, E. Joseph, G. Sciutto, R. Mazzeo, Acc. Chem. Res. 43, 792-801 (2010)
7. M. Spring, C. Ricci, D.A. Peggie, S.G. Kazarian, Anal. Bioanal. Chem. 392, 37-45 (2008)

8. M. Odlyha, Thermochim. Acta 269-270, 705-727 (1995)

9. R. Ploeger, D. Scalarone, O. Chiantore, J. Cult. Heritage 11, 1541 (2011)

10. M.R. Derrick, D. Stulik, J.M. Landry, Infrared Spectroscopy in Conservation Science (The Getty Conservation Institute, Los Angeles, 1999)

11. R. Mazzeo, S. Prati, M. Quaranta, J.E. Kendix, M. Galeotti, Anal. Bioanal. Chem. 392, 65-76 (2008)

12. R. Mazzeo, E. Joseph, S. Prati, A. Millemaggi, Anal. Chim. Acta 599, 107-117 (2007)

13. A. Jurado-López, M.D.L. de Castro, Anal. Bioanal. Chem. 380, 706-711 (2004)

14. A. Miklós, S. Schäfer, P. Hess, Photoacoustic spectroscopy, theory, in Encyclopedia of Vibrational Spectroscopy, ed. by P. Griffiths, J. Chalmers (Wiley, Chichester, 2001)

15. J.F. McClelland, R.W. Jones, S.J. Bajic, in Handbook of Vibrational Spectroscopy, vol. 2, ed. by J.M. Chalmers, P.R. Griffiths (Wiley, London, 2002), pp. 1231-1251

16. M. Doerner, The Materials of the Artist and Their Use in Painting: with Notes on the Techniques of the Old Masters, revised edn (Harcourt, New York, 1984), 